\title{
Adjuvant therapy for primary GIST: is longer really better? A narrative review
}

\author{
Pamela W. Lu ${ }^{1}$, Chandrajit P. Raut ${ }^{1,2,3}$ \\ ${ }^{1}$ Division of Surgical Oncology, Brigham and Women's Hospital, Boston, MA, USA; ${ }^{2}$ Center for Sarcoma and Bone Oncology, Dana-Farber Cancer \\ Institute, Boston, MA, USA; ${ }^{3}$ Harvard Medical School, Boston, MA, USA \\ Contributions: (I) Conception and design: All authors; (II) Administrative support: None; (III) Provision of study materials or patients: None; (IV) \\ Collection and assembly of data: All authors; (V) Data analysis and interpretation: None; (VI) Manuscript writing: All authors; (VII) Final approval of \\ manuscript: All authors. \\ Correspondence to: Chandrajit P. Raut, MD, MSc. Sarcoma Suite, Dana 2, Dana-Farber Cancer Institute, 450 Brookline Avenue, Boston, MA 02115, \\ USA. Email: craut@bwh.harvard.edu.
}

\begin{abstract}
Objective: Review and critically evaluate the landmark studies that examined the role of adjuvant imatinib in resectable gastrointestinal stromal tumor (GIST) to provide updated recommendations regarding duration of therapy.
\end{abstract}

Background: GISTs are the most common mesenchymal tumors of the gastrointestinal tract. Surgery remains the mainstay of potentially curative treatment, but recurrence rates after macroscopically complete resection remain high. Improved understanding of the molecular behavior of GISTs and the success of the tyrosine kinase inhibitor imatinib in the treatment of metastatic GIST led to the investigation of imatinib as adjuvant therapy in several clinical trials. Adjuvant imatinib has been shown to significantly improve recurrence-free survival rates in patients with intermediate- or high-risk tumors and, with longer duration, overall survival in patients with high-risk tumors. The current practice for patients who have had preoperative imatinib or are otherwise considered to be at intermediate to high risk of recurrence after resection is to be treated with adjuvant imatinib for 3 years. In this context, the question of whether further prolonging the course of adjuvant imatinib beyond the current standard is beneficial to patients who have undergone complete macroscopic resection of GIST tumors arises.

Methods: We critically reviewed the data from landmark studies regarding the use of imatinib treatment in resectable GIST.

Conclusions: Early imatinib discontinuation rates have remained a persistent challenge, and the benefit of further increasing duration of adjuvant treatment remains unclear. The choice to pursue longer term adjuvant imatinib therapy should depend on a careful risk-benefit analysis and discussion between patients and their treating clinicians.

Keywords: Gastrointestinal stromal tumor (GIST); imatinib; adjuvant therapy

Received: 26 February 2021; Accepted: 30 June 2021; Published: 20 August 2021.

doi: 10.21037 /gist-21-4

View this article at: https://dx.doi.org/10.21037/gist-21-4

\section{Introduction}

Gastrointestinal stromal tumors (GISTs) are rare neoplasms and account for only an estimated $0.1 \%$ to $3 \%$ of gastrointestinal malignancies with approximately 5,000 cases diagnosed in the United States annually $(1,2)$.
Despite its relative rarity, GISTs are the most common mesenchymal tumor of the gastrointestinal tract, and the rate of diagnosis has been increasing in recent years $(3,4)$. Though previously a poorly understood disease, discoveries leading to the identification of the KIT and PDGFRA activating mutations of receptor tyrosine kinases 
in many GISTs have resulted in successful clinical use of tyrosine kinase inhibitors in treating this neoplasm, particularly in the metastatic setting (1). Surgery remains the mainstay of treatment for resectable primary GISTs but unfortunately, the recurrence rate even after a macroscopically complete resection remained high at over $40 \%$ with an unacceptably high mortality rate $(5,6)$. This has improved with the use of adjuvant therapy with the tyrosine kinase inhibitor imatinib; however the optimal duration of adjuvant imatinib treatment remains unclear $(6,7)$. Here, we critically examine the current data from landmark studies regarding adjuvant imatinib treatment for GIST. We present the following article in accordance with the narrative review reporting checklist (available at https://dx.doi.org/10.21037/gist-21-4).

\section{Impact of adjuvant treatment on progression-free survival}

The first study launched evaluating the use of adjuvant imatinib for GIST was the American College of Surgeons Oncology Group (ACOSOG) Z9000 trial conducted between 2001 and 2003 (8). In this phase II, single-arm study, 106 patients who had undergone macroscopically complete tumor resection but were considered high risk for recurrence (based on tumor size $>10 \mathrm{~cm}$, tumor rupture, or up to 4 peritoneal implants) were placed on a regimen of imatinib of $400 \mathrm{mg}$ daily for 1 year. The primary endpoint was overall survival (OS) of the patient cohort compared to that of historical records for control, and secondary endpoints included recurrence-free survival (RFS) and safety. The 5 -year OS of $83 \%$ with 1 year of adjuvant therapy compared favorably with the historical 5 -year OS of $35 \%$ seen with surgery alone.

In the ACOSOG Z9001 randomized, double-blinded, phase III trial, patients who underwent a macroscopically complete resection of a GIST $\geq 3 \mathrm{~cm}$ in size and positive for KIT were randomized to receive 1 year of either $400 \mathrm{mg} /$ day of imatinib (359 patients) or placebo (354 patients) between 2002 and 2007 (9). There was significant improvement in 1 -year RFS in the imatinib arm compared to the placebo arm, $98 \%$ vs. $83 \%$. Interestingly, the RFS curves between groups appeared to follow similar slopes, though the experimental group's curve was offset by approximately 18 months $(7,9)$. There was no significant difference in OS between groups at one year $(99.2 \%$ treatment $v$ s. $99.7 \%$ placebo), which was not surprising given the crossover design.
This trial confirmed that adjuvant treatment with imatinib after resection of KIT-positive GIST delayed recurrence. However, it raised the question of whether adjuvant therapy truly improved long-term outcomes or simply delayed them. Furthermore, the rates of early treatment discontinuation were $27 \%$ in the imatinib arm and $25 \%$ in the placebo arm. Nonetheless, based on these reported results, in 2008 the Food and Drug Administration approved the use of imatinib as adjuvant treatment for patients with KIT positive GIST who have undergone surgical resection (7). Findings of these trials are summarized in Table 1.

\section{Impact of adjuvant treatment on overall survival}

The Scandinavian Sarcoma Group XVIII/Arbeitsgemeinschaft Internistische Onkologie (SSGXVIII/AIO) randomized, open-label trial enrolled a total of 400 patients between 2004 and 2008 to receive either 1 year or 3 years of adjuvant imatinib after macroscopically complete resection of KIT-positive GIST with high risk of recurrence based on the NIH consensus criteria (10). In 2012, the initial results were published showing improved 5-year RFS in the 3 -year arm at $65.6 \%$ compared to $47.9 \%$ seen with 1 year of adjuvant treatment. This study also demonstrated, for the first time, significant improvement in 5 -year OS with the longer treatment course, $92.0 \% v s$. $81.7 \%$, respectively. These findings supported prolonged treatment for improvement in both RFS and OS. Importantly, however, there was no significant difference in disease-specific survival found between groups, though this trended in favor of the 3-year arm. Of note, while only $12.6 \%$ of patients in the 1-year group ended treatment early for reasons other than recurrence, $25.8 \%$ of patients in the 3 -year treatment group did not complete the prescribed course.

In 2020, the authors reported 10-year follow up data which demonstrated continued benefit of the longer adjuvant treatment course. The 10-year RFS for the 3-year treatment group was $52.5 \%$ compared to $41.8 \%$ for those in the 1-year group, and 10-year OS was 79.0\% compared to $65.3 \%$ for the 3 - and 1 -year treatment groups, respectively (11). While disease-specific survival results were not reported, the authors reported that over $80 \%$ of patients who died in both arms had confirmed metastatic disease. These data confirmed that a longer course of adjuvant treatment had superior efficacy in patients at a high risk of recurrence after resection. 


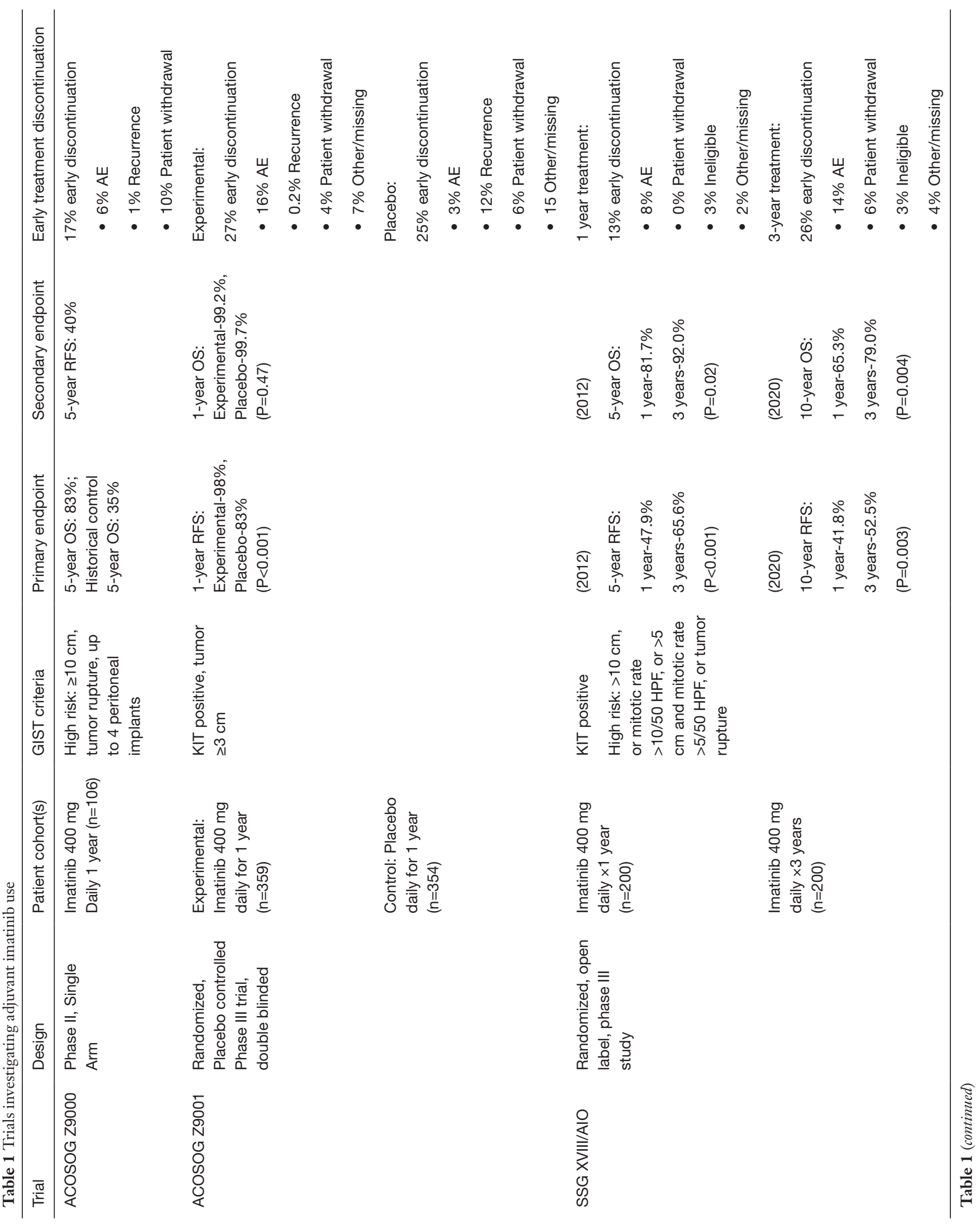




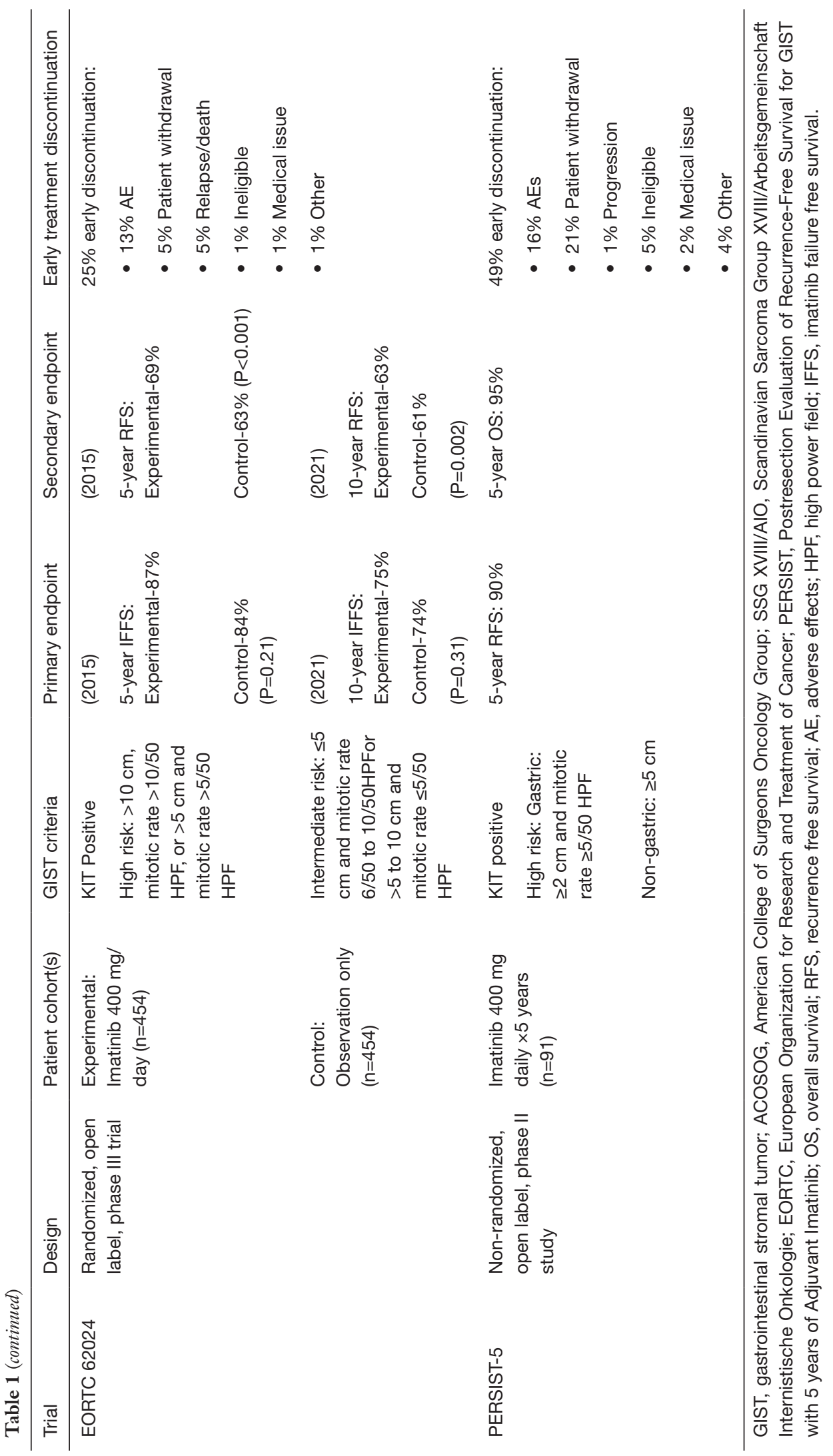


Another manuscript from this study focused on the impact of mutation status on outcome. Longer duration adjuvant therapy had greater benefit in patients with KIT exon 11 deletion or insertion/deletion mutations (12). No benefits were seen in patients with KIT exon 9, PDGFRA, or other mutations.

\section{Timing of imatinib on second-line therapy}

The European Organization for Research and Treatment of Cancer (EORTC) launched a randomized, open label, phase III trial (EORTC 62024) across 12 countries between 2004 and 2008; 454 patients were randomized to the observational arm and 454 patients to the treatment arm, where 2 years of imatinib $400 \mathrm{mg} /$ day after macroscopically complete resection was prescribed (13). Patients had intermediate or high risk of recurrence based on tumor size and mitoses per high power field described by the 2002 NIH consensus guidelines (14). The initial primary endpoint of OS was changed to "imatinib failure-free survival" (IFFS). No difference in IFFS was found between groups. The RFS in the treatment arm was significantly higher than the observation arm, with $84 \%$ vs. $66 \%$ at 3 years and $69 \%$ vs. $63 \%$ at 5 years, respectively. These findings suggested that while RFS was prolonged with adjuvant treatment (as seen in the other trials), there was no difference in time to initiation of second line of therapy if imatinib treatment was initiated immediately after resection or later after initial recurrence. However, a subset analysis in high-risk patients suggested a trend favoring longer IFFS with adjuvant therapy. Discontinuation rates were high in this study, $25 \%$, consistent with the prior trials.

In 2021, the authors published 10-year follow-up data (15). The RFS benefits were sustained with adjuvant therapy, and there was no difference in OS or IFFS. Once again, there was a trend in IFFS in high-risk patients favoring the adjuvant therapy arm (69\% vs. 61\%), but this did not reach statistical significance.

One of the key results from this study suggested that a 2-year course of adjuvant therapy was not of sufficient duration to result in improved OS, as opposed to 3 years of adjuvant imatinib in the Scandinavian/German study. Secondly, though the study was negative with respect to the primary endpoint IFFS across the entire cohort, the trend favoring adjuvant therapy in the high-risk subset of patients suggested that those at highest risk of recurrence may be the ones most likely to benefit from adjuvant imatinib.

\section{Duration of therapy}

The question of the benefit of even longer duration of adjuvant therapy was then addressed by the Postresection Evaluation of Recurrence-Free Survival for GIST with 5 years of Adjuvant Imatinib (PERSIST-5) trial, a prospective, single-arm phase II clinical trial (16). A total of 91 patients were enrolled between 2009 and 2016 after a macroscopically complete resection of KIT positive primary GIST with intermediate or high risk of recurrence based on the Miettinen and Lasota criteria (3) to receive $400 \mathrm{mg} /$ day of imatinib for a total duration of 5 years. The primary endpoint was RFS, with OS, safety and tolerability defined as secondary endpoints. Median duration of treatment was 55.1 months, and the 5-year RFS was $90 \%$. Of the 7 patients who had disease recurrence, only 1 recurred while receiving imatinib treatment; this patient had a PDGFRA D $842 \mathrm{~V}$ mutation. The 5-year OS was high at $95 \%$. This study cohort demonstrated a high early therapy discontinuation rate, with $49 \%$ of patients discontinuing therapy prior to completion. The most frequent reason for discontinuation was patient choice (21\%), followed by adverse events (17\%). The 6 patients who recurred after stopping imatinib (1 after completing 5 years of therapy, 5 after early discontinuation) recurred between 7 and 24 months after their final adjuvant dose.

\section{Discussion}

There is no doubt that imatinib has dramatically changed the landscape for management of GIST recurrence. However, questions regarding the optimal use of this adjuvant treatment remain.

Results from the studies outlined above support the fact that imatinib delays recurrence, and longer treatment duration results in a longer delay, essentially "kicking the can down the road." Furthermore, longer therapy (at least 3 years) is associated with improved OS, as confirmed by early and longer-term follow-up from the SSGXVIII/AIO trial. However, DSS was not prolonged in the initial report, but with prolonged follow-up, the overwhelming majority of patients who died had confirmed metastatic disease. The EORTC 62024 showed that adjuvant imatinib may result in better long-term outcomes (change to second line therapy) in high-risk patients than waiting until recurrence to start imatinib.

Longer treatment, however, does come at a price of increased patient discontinuation rates-in the PERSIST-5 
Table 2 Ongoing trials

\begin{tabular}{|c|c|c|c|c|c|}
\hline Trial & Design & Patient cohort(s) & Gist criteria & Primary endpoint & Secondary endpoint \\
\hline NCT02260505 & $\begin{array}{l}\text { Randomized, } \\
\text { open label, Phase } \\
\text { III trial }\end{array}$ & $\begin{array}{l}\text { No intervention: } \\
\text { Interruption of imatinib } \\
\text { after } 3 \text { years of adjuvant } \\
\text { treatment }\end{array}$ & $\begin{array}{l}>35 \% \text { risk of } \\
\text { recurrence based on } \\
\text { NCCN criteria }\end{array}$ & $\begin{array}{l}\text { Disease-free } \\
\text { survival }\end{array}$ & $\begin{array}{l}\text { OS, Time to secondary } \\
\text { resistance, \% patients } \\
\text { in complete response, } \\
\text { Frequency of AEs, } \\
\text { Patient QoL }\end{array}$ \\
\hline NCT02413736 & $\begin{array}{l}\text { Randomized, } \\
\text { open label, Phase } \\
\text { III trial }\end{array}$ & $\begin{array}{l}\text { Experimental: Imatinib } \\
400 \text { mg/day for } \\
24 \text { months }\end{array}$ & $\begin{array}{l}\text { Non-gastric GIST: } \\
\text { mitotic count } \\
>5 / 50 \text { HPFs or tumor } \\
\text { rupture }\end{array}$ & $\begin{array}{l}\text { Disease-free } \\
\text { survival }\end{array}$ & $\begin{array}{l}\text { OS, GIST-specific } \\
\text { survival, AEs }\end{array}$ \\
\hline
\end{tabular}

GIST, gastrointestinal stromal tumor; NCCN, National Comprehensive Cancer Network; OS, overall survival; AE, adverse effects; QoL, quality of life; HPF, high power fields.

trial, nearly half of the patients did not complete the initially prescribed 5-year course, and the rate of discontinuation remained stable throughout the study period (16). Although imatinib is a relatively well tolerated adjuvant treatment, virtually all patients across many different studies experienced at least one drug related AE (17). Fortunately, the majority of AEs were grade 1 or 2 and not severe (17). And despite the results of the SSGXVIII/AIO data available confirming improved OS, patients in the PERSIST-5 trial still opted to discontinue therapy at a high rate.

The data from prior studies must be viewed within the context of each study design's limitations. As the knowledge regarding GIST mutations and stratification of recurrence risk has grown markedly over the past two decades, it is important to consider the fact that many of these studies were performed as the understanding of GIST tumor biology and behavior was evolving. For example, in the ACOSOC Z9001 study patients with tumors $\geq 3 \mathrm{~cm}$ were included despite the fact that some may not have met criteria for intermediate or high risk of recurrence (9). In studies that did discriminate between strata of recurrence risk, the criteria used to define them as such also varied: for example, the SSGVXVIII/AIO study utilized the NIH criteria (14), whereas the PERSIST-5 trial followed the Miettinen and Lasota criteria $(3,16)$. Similarly, none of the studies excluded patients with tumors harboring mutations later discovered to confer imatinib resistance which can skew results, based on the available data at the time the trials were designed $(7,10)$. Another factor that must be considered is the potential effects on developing imatinib resistance in the context of prolonged maintenance treatment, a phenomenon that has been seen with patients with metastatic disease but has not been well studied in the adjuvant treatment setting (6). The EORTC 62024 results (trend favoring adjuvant imatinib in high-risk patients) suggested that early imatinib exposure does not necessarily increase the potential for faster development of imatinib resistance when resumed at the time of recurrence.

Currently, the National Comprehensive Cancer Network (NCCN) guidelines recommend consideration of adjuvant treatment only for patients with resected intermediate or high risk disease with a standard duration of 3 years, and while the ability to prolong treatment in certain cases is available in the US, there is little flexibility in other international guidelines (18).

Looking forward, the critical trials needed to further address the question of utility of prolonged adjuvant imatinib treatment are underway (Table 2). NCT02260505, or Efficiency of Imatinib Treatment Maintenance or Interruption After 3 Years of Adjuvant Treatment in Patients With Gastrointestinal Stromal Tumours (GIST) (ImadGist) opened in October 2014 and is ongoing. This French Sarcoma Group randomized discontinuation trial aims to evaluate the effect of prolonged adjuvant imatinib treatment of 6 years compared to the current 
standard of 3 years in patients with estimated $>35 \%$ risk of recurrence based on NCCN risk classification after gross resection (19). The estimated completion date for this trial is December 2022. Another trial, NCT02413736, or the Three versus Five Years of Adjuvant Imatinib as Treatment of Patients with Operable GIST With a High Risk For Recurrence: a Randomized Phase III Study began enrollment in May 2015 (20). In this Scandinavian Sarcoma Group trial, patients who completed 36 months of adjuvant imatinib after resection of a GIST at high risk of recurrence (non-gastric GIST mitotic count $>5 / 50 \mathrm{HPFs}$ or tumor rupture, or gastric GIST mitotic count $>10 / 50$ HPFs) will be 1:1 randomized to either continue imatinib for 2 more years, or stop. The trial has reported an estimated primary completion date in May 2028. The results of these studies will help better define optimal adjuvant treatment duration length of patients who have undergone macroscopic resection of high risk of recurrence.

For these longer duration trials, early discontinuation rates should be monitored given the high drop-out rates seen in the completed studies. Patients should be followed closely during the first 2 years after stopping adjuvant treatment given risk of recurrence during that time frame. Early detection of recurrence is critical for this patient population as prior studies have suggested that rescue therapy with imatinib after recurrence can still be effective. In those where rescue therapy with standard dosing is not effective, high dose regimens can also be trialed, or second and third-line agents (6). Current NCCN guidelines recommend follow-up every 3 to 6 months for 5 years after resection regardless of use of adjuvant treatment, with every 3 months for those who are high risk for recurrence (18).

Several questions are unanswered. For patients with imatinib insensitive mutations such as KIT exon 9 mutations, it is unclear if higher doses of imatinib confers the same sort of benefit in the adjuvant setting as it does in the metastatic setting (21). Likewise for patients with the imatinib insensitive mutation PDGFRA D $842 \mathrm{~V}$, the efficacy of the TKI avapritinib, recently approved by the FDA and EMA for unresectable or metastatic PDGFRA D842V GIST, in the adjuvant setting is unknown (22). It is also unclear if imatinib doses lower than the standard $400 \mathrm{mg} /$ day offer any efficacy in the adjuvant setting. Finally, while patients with ruptured GIST were included in some of the trials, it is unclear if they would derive benefit from longer duration, or even indefinite, imatinib therapy.

\section{Conclusions}

Ultimately, like most treatment decisions, the choice to pursue longer term adjuvant imatinib therapy should depend on a careful risk-benefit analysis and discussion between patients and their treating clinicians. Adjuvant treatment in patients with imatinib-sensitive mutations with high-risk of recurrence has been shown to delay relapse (with any duration of therapy evaluated). The benefit in overall survival is only seen with at least 3 years of adjuvant therapy, and that was from a single trial enrolling essentially only high-risk patients. The sustained benefit in delaying time to second-line therapy may also only be realized in high-risk patients. The burden of committing to longer term treatment and facing the risk of associated AEs may be beneficial only in a certain subset of patients, and thus use of adjuvant imatinib should be limited to those most likely to benefit. When analyzing each patient's risk of recurrence, factors such as mutational status and history of tumor rupture should be carefully considered in addition to the classically described pathologic criteria, as these can all point to a higher recurrence risk. While a new standard may be set after the results of the NCT02413736 and NCT02260505 trials are reported, current best practices for extending adjuvant treatment beyond three years relies on thorough counseling and individualized shared decisionmaking between patient and provider, as data regarding benefit of extended duration are limited to results of a single phase II trial.

\section{Acknowledgments}

Funding: None.

\section{Footnote}

Provenance and Peer Review: This article was commissioned by the Guest Editors (Bernd Kasper and Eva Wardelmann) for the series "Gastrointestinal Stromal Tumors" published in Gastrointestinal Stromal Tumor. The article has undergone external peer review.

Reporting Checklist: The authors have completed the narrative review reporting checklist. Available at https:// dx.doi.org/10.21037/gist-21-4

Conflicts of Interest: Both authors have completed the 
ICMJE uniform disclosure form (available at https://dx.doi. org/10.21037/gist-21-4). The series "Gastrointestinal Stromal Tumors" was commissioned by the editorial office without any funding or sponsorship. The authors have no other conflicts of interest to declare.

Ethical Statement: The authors are accountable for all aspects of the work in ensuring that questions related to the accuracy or integrity of any part of the work are appropriately investigated and resolved.

Open Access Statement: This is an Open Access article distributed in accordance with the Creative Commons Attribution-NonCommercial-NoDerivs 4.0 International License (CC BY-NC-ND 4.0), which permits the noncommercial replication and distribution of the article with the strict proviso that no changes or edits are made and the original work is properly cited (including links to both the formal publication through the relevant DOI and the license). See: https://creativecommons.org/licenses/by-nc-nd/4.0/.

\section{References}

1. Hong NJL, Raut CP. Gastrointestinal Stromal Tumors. In: Zinner MJ, Ashley SW, Hines OJ, editors. Maingot's Abdominal Operations, 13e. New York, NY: McGraw-Hill Education; 2019.

2. American Cancer Society. Key Statistics for Gastrointestinal Stromal Tumors American Cancer Society2019. Available online: https://www.cancer. org/cancer/gastrointestinal-stromal-tumor/about/keystatistics.html

3. Miettinen M, Lasota J. Gastrointestinal stromal tumors: pathology and prognosis at different sites. Semin Diagn Pathol 2006;23:70-83.

4. Patel N, Benipal B. Incidence of Gastrointestinal Stromal Tumors in the United States from 2001-2015: A United States Cancer Statistics Analysis of 50 States. Cureus 2019;11:e4120.

5. DeMatteo RP, Lewis JJ, Leung D, et al. Two hundred gastrointestinal stromal tumors: recurrence patterns and prognostic factors for survival. Ann Surg 2000;231:51-8.

6. Keung EZ, Raut CP. Management of Gastrointestinal Stromal Tumors. Surg Clin North Am 2017;97:437-52.

7. Keung EZ, Raut CP, Rutkowski P. The Landmark Series: Systemic Therapy for Resectable Gastrointestinal Stromal Tumors. Ann Surg Oncol 2020;27:3659-71.

8. DeMatteo RP, Ballman KV, Antonescu CR, et al. Long- term results of adjuvant imatinib mesylate in localized, high-risk, primary gastrointestinal stromal tumor: ACOSOG Z9000 (Alliance) intergroup phase 2 trial. Ann Surg 2013;258:422-9.

9. Dematteo RP, Ballman KV, Antonescu CR, et al. Adjuvant imatinib mesylate after resection of localised, primary gastrointestinal stromal tumour: a randomised, doubleblind, placebo-controlled trial. Lancet 2009;373:1097-104.

10. Joensuu H, Eriksson M, Sundby Hall K, et al. One vs three years of adjuvant imatinib for operable gastrointestinal stromal tumor: a randomized trial. JAMA 2012;307:1265-72.

11. Joensuu H, Eriksson M, Sundby Hall K, et al. Survival Outcomes Associated With 3 Years vs 1 Year of Adjuvant Imatinib for Patients With High-Risk Gastrointestinal Stromal Tumors: An Analysis of a Randomized Clinical Trial After 10-Year Follow-up. JAMA Oncol 2020;6:1241-6.

12. Joensuu H, Wardelmann E, Sihto H, et al. Effect of KIT and PDGFRA Mutations on Survival in Patients With Gastrointestinal Stromal Tumors Treated With Adjuvant Imatinib: An Exploratory Analysis of a Randomized Clinical Trial. JAMA Oncol 2017;3:602-9.

13. Casali PG, Le Cesne A, Poveda Velasco A, et al. Time to Definitive Failure to the First Tyrosine Kinase Inhibitor in Localized GI Stromal Tumors Treated With Imatinib As an Adjuvant: A European Organisation for Research and Treatment of Cancer Soft Tissue and Bone Sarcoma Group Intergroup Randomized Trial in Collaboration With the Australasian Gastro-Intestinal Trials Group, UNICANCER, French Sarcoma Group, Italian Sarcoma Group, and Spanish Group for Research on Sarcomas. J Clin Oncol 2015;33:4276-83.

14. Fletcher CD, Berman JJ, Corless C, et al. Diagnosis of gastrointestinal stromal tumors: A consensus approach. Hum Pathol 2002;33:459-65.

15. Casali PG, Le Cesne A, Velasco AP, et al. Final analysis of the randomized trial on imatinib as an adjuvant in localized gastrointestinal stromal tumors (GIST) from the EORTC Soft Tissue and Bone Sarcoma Group (STBSG), the Australasian Gastro-Intestinal Trials Group (AGITG), UNICANCER, French Sarcoma Group (FSG), Italian Sarcoma Group (ISG), and Spanish Group for Research on Sarcomas (GEIS) 弥. Ann Oncol 2021;32:533-41.

16. Raut CP, Espat NJ, Maki RG, et al. Efficacy and Tolerability of 5-Year Adjuvant Imatinib Treatment for Patients With Resected Intermediate- or High-Risk Primary Gastrointestinal Stromal Tumor: The PERSIST-5 
Clinical Trial. JAMA Oncol 2018;4:e184060.

17. Thanopoulou E, Judson I. The safety profile of imatinib in CML and GIST: long-term considerations. Arch Toxicol 2012;86:1-12.

18. National Comprehensive Cancer Network.

Gastrointestinal Stromal Tumors (GIST) Version 1.2021:

NCCN.org; 2020. Available online: https://www.nccn.org/ professionals/physician_gls/pdf/gist.pdf.

19. ClinicalTrials.gov. Efficiency of Imatinib Treatment Maintenance or Interruption After 3 Years of Adjuvant Treatment in Patients With Gastrointestinal Stromal Tumours (GIST) (ImadGist): NCT02260505 2020. Available online: https://clinicaltrials.gov/ct2/show/ NCT02260505.

doi: 10.21037/gist-21-4

Cite this article as: Lu PW, Raut CP. Adjuvant therapy for primary GIST: is longer really better? A narrative review. Gastrointest Stromal Tumor 2021;4:4.
20. ClinicalTrials.gov. Three Versus Five Years of Adjuvant Imatinib as Treatment of Patients With Operable GIST: NCT02413736 2020. Available online: https:// clinicaltrials.gov/ct2/show/NCT02413736.

21. Gastrointestinal Stromal Tumor Meta-Analysis Group (MetaGIST). Comparison of two doses of imatinib for the treatment of unresectable or metastatic gastrointestinal stromal tumors: a meta-analysis of 1,640 patients. J Clin Oncol 2010;28:1247-53.

22. Jones RL, Serrano C, von Mehren M, et al. Avapritinib in unresectable or metastatic PDGFRA D842V-mutant gastrointestinal stromal tumours: Long-term efficacy and safety data from the NAVIGATOR phase I trial. Eur J Cancer 2021;145:132-42. 